\title{
Optimisation of Three-Dimensional Imaging of the Breast Region with 3-D Laser Scanners
}

\author{
Maximilian EDER ${ }^{\star a}$, Fee ARMBRECHT ${ }^{\mathrm{a}}$, Stefan RAITH ${ }^{\mathrm{a}}$, Alexander VOLF ${ }^{\mathrm{a}}$, \\ Alexander ZIMMERMANN ${ }^{b}$, Nikolaos A. PAPADOPULOS ${ }^{a}$, and Laszlo KOVACS ${ }^{a}$ \\ ${ }^{a}$ Research Group Computer Aided Plastic Surgery - CAPS, \\ Clinic for Plastic Surgery and Hand Surgery, Technical University Munich, Germany; \\ ${ }^{\mathrm{b}}$ Clinic for Vascular Surgery, Technical University Munich, Germany
}

\begin{abstract}
Introduction: Only few objective measuring methods in plastic surgery exist, which allow to display the human body in its entire complex surface. Therefore, the success of breast surgery is often the result of the surgeon's skills for visual imaging and assessing the breast. Currently, 2-D photos are the most commonly used method for documentation and planning the surgical treatment. But these contain numerous errors due to the perspective distortions. In addition, they lack metric and 3-D information. 3-D surface imaging for breast surgery gain more and more importance in the last few years. The primary objective of the present study was to evaluate the quality, i.e. the reproducibility and accuracy of the 3-D images of the breast region we imaged with the laser linear scanner under various trial set-ups and to make definitive decisions about the best imaging techniques about methods of explorative and confirmatory statistics.
\end{abstract}

Materials and Methods: Imaging of the 3-D shape of the breast region was carried out with the Minolta Vivid 910 scanner (Konica-Minolta Co, Ltd, Osaka, Japan) and evaluated with appropriate software (RapidForm 2002 PP1 SP1 software, INUS Technology, Inc, Seoul, South Korea). Avoiding the influence of biotic factors, such as mobility, we tested the most favorable imaging technology on two life-size dummy models, one with smaller and one with larger breasts (Fig. 1A, B), for scanner-related factors such as the scanner position in comparison with the torso and the number of scanners and single shots (Fig. 1C, D). The influence of different factors of the breast region, such as different breast shapes or premarking of anatomic landmarks, was also first investigated on dummies. The findings from the dummy models were then compared with investigations on test persons (Fig. 1E, $\mathrm{F}$ ), and the accuracy of measurements on the virtual models was compared with a coincidence analysis of the manually measured values.

Results: The best precision and accuracy of breast region measurements were achieved when landmarks were marked before taking the shots and when shots at 30 degrees left and 30 degrees right, relative to the sagittal line, were taken with 2 connected scanners mounted with a 10-degree upward angle (TABLE 1). However, the precision of the measurements on test persons was significantly lower than those measured on dummies (Fig. 2 and 3). The submammary region of very large breasts is very difficult to image, which infects the precision. The accuracy primarily depends of the correct setting for 3-D imaging.

Conclusion: 3-D surface imaging of the breast region using 3-D surface imaging is possible with an acceptable degree of accuracy and reproducibility. The standardisation of the imaging position and the scanning technique is decisive to guarantee the reproducibility of the method. Further developments of the scanner system or application of scanners, which allow faster recording from several angles with automatic image recording, could improve the quality of these shots. 3-D surface imaging of the breast region could become an important instrument for the evaluation, planning and even for simulating breast surgery.

Keywords: breast, 3-D surface imaging, breast surgery 


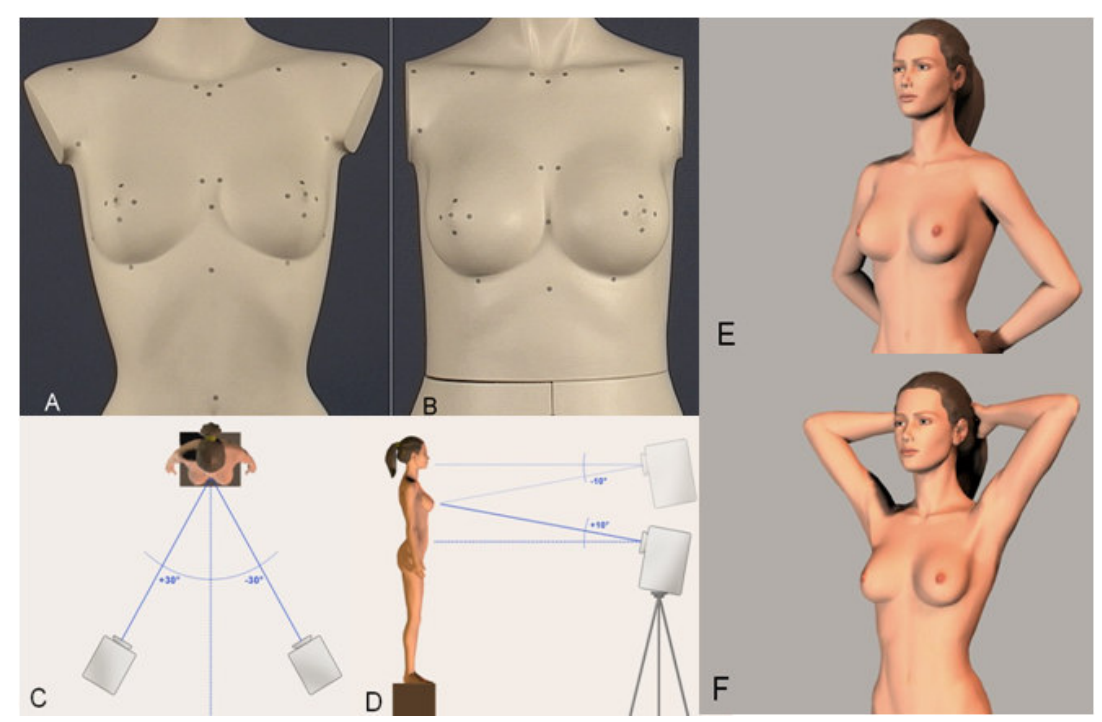

Fig. 1: Investigations of the dummies and application and validation of the findings on test persons

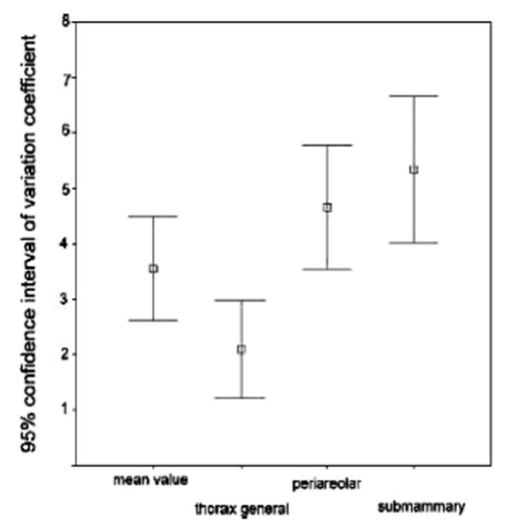

Fig. 2: The precision of the measurements on the 3Dimages of the breast region.

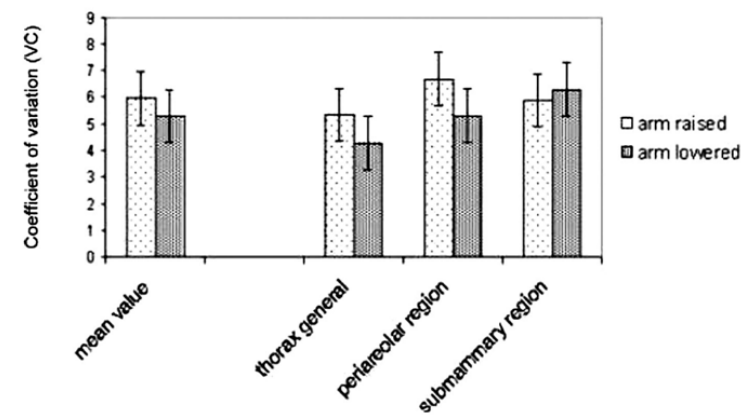

Fig. 3: The precision of the measurements in connection with the number of the scanners used and the single shots. Precision of the measurements according to the anatomic regions:mean value of the coefficient of variation (VC) for all test persons.

\begin{tabular}{|c|c|c|c|c|c|c|c|c|c|c|c|c|}
\hline & \multicolumn{6}{|c|}{ Without Marked Landmarks } & \multicolumn{6}{|c|}{ With Marked Landmarks } \\
\hline & \multicolumn{3}{|c|}{ Dummy $A^{*}$} & \multicolumn{3}{|c|}{ Dummy B ${ }^{t}$} & \multicolumn{3}{|c|}{ Dummy A } & \multicolumn{3}{|c|}{ Dummy B } \\
\hline & Mean & SEM & No. & Mean & SEM & No. & Mean & SEM & No. & Mean & SEM & No. \\
\hline \multicolumn{13}{|c|}{1 Scanner and 2 shots } \\
\hline Thorax gen. & 3.60 & 0.29 & 144 & 5.12 & 0.47 & 144 & 1.11 & 0.10 & 144 & 2.11 & 0.38 & 144 \\
\hline Periareolar & 4.86 & 0.43 & 111 & 7.04 & 0.59 & 111 & 2.39 & 0.19 & 111 & 2.76 & 0.25 & 111 \\
\hline Submammary & 4.01 & 0.53 & 60 & 9.01 & 1.35 & 60 & 3.57 & 0.59 & 60 & 2.61 & 0.41 & 60 \\
\hline \multicolumn{13}{|c|}{1 Scanner and 3 shots } \\
\hline Thorax gen. & 390 & 0.31 & 144 & 2.99 & 0.23 & 144 & 2.30 & 0.24 & 144 & 1.24 & 0.14 & 144 \\
\hline Periareolar & 6.67 & 0.61 & 111 & 3.37 & 0.26 & 111 & 3.43 & 0.30 & 111 & 1.87 & 0.17 & 111 \\
\hline Submammary & 5.99 & 0.90 & 60 & 3.37 & 0.42 & 60 & 4.60 & 0.76 & 60 & 2.01 & 0.44 & 60 \\
\hline \multicolumn{13}{|c|}{2 Scanners and 2 shots } \\
\hline Thorax gen. & 3.58 & 0.27 & 144 & 2.46 & 0.20 & 144 & 1.19 & 0.11 & 144 & 0.95 & 0.11 & 144 \\
\hline Periareolar & 5.00 & 0.45 & 111 & 3.42 & 0.29 & 111 & 3.14 & 0.37 & 111 & 1.51 & 0.13 & 111 \\
\hline Submammary & 4.15 & 0.53 & 60 & 3.80 & 0.48 & 60 & 6.45 & 1.49 & 60 & 1.62 & 0.24 & 60 \\
\hline
\end{tabular}

